\title{
Special issue on intelligent computing methodologies in machine learning for IoT applications
}

\author{
Jinghua Zhao' ${ }^{1}$ Junyu Xuan ${ }^{2}$ \\ Published online: 12 June 2021 \\ (C) The Author(s), under exclusive licence to Springer-Verlag London Ltd., part of Springer Nature 2021
}

Machine learning is to design and analyze algorithms that allow computers to "learn" automatically, and allows machines to establish rules from automatically analyzing data and using them to predict unknown data. Traditional machine learning approach is difficult to meet the needs of internet of things (IoT) only through its outdated process starting from problem definition, appropriate information collection, and ending with model development and results verification. But, however, recent scenario has dramatically changed due to the development of artificial intelligence (AI) and high-speed computing performance. Machine learning has been applied to solve complex problems in human society for years, and the success of machine learning is because of the support of computing capabilities as well as the sensing technology. An evolution of artificial intelligence and intelligent computing approaches will soon cause considerable impacts to the field. Search engines, image recognition, biometrics, speech and handwriting recognition, natural language processing, and even medical diagnostics and financial credit ratings are all common examples. It is clear that many challenges will be brought to publics as the artificial intelligence infiltrates into our world, and more specifically, our lives.

The submitted manuscripts were reviewed by experts from both academia and industry. After two rounds of reviewing, the highest quality manuscripts were accepted for this special issue. This special issue will be published by Neural Computing and Applications as special issues.

Jinghua Zhao

zhaojinghua@usst.edu.cn

1 School of Business, University of Shanghai for Science and Technology, Shanghai 200093, China

2 Faculty of Engineering and Information Technology, Australia Artificial Intelligence Institute, University of Technology Sydney, 15 Broadway, Sydney, NSW 2007, Australia
Totally, 14 papers are suggested to EiC for acceptance. The selected papers are summarized as follows.

Sun et al. [1] combine the concept of deep learning to carry out algorithm innovation in the research. Li et al. [2] propose a fast convolutional neural network image segmentation method to segment the indoor scene image and construct the fast fully convolutional networks (FFCN) for indoor scene image segmentation and use inter-layer fusion to reduce the amount of network calculation parameters and avoid the loss of picture feature information by continuous convolution. Cai et al. [3] propose a new convolution kernel, which can effectively detect the corresponding features with different transformations by actively transforming the relative positions of the connections in the convolution kernel. Zhou et al. [4] design and implement a bank CRM system based on decision tree algorithm. Sun et al. [5] apply convolutional neural network to CT image classification, so as to speed up CT image classification and improve the accuracy of CT image classification and so as to reduce the workload of doctors and improve work efficiency. Sun et al. [6] study the neural network control system of cooperative robot based on genetic algorithm. Li et al. [7] combine machine learning technology to construct a traffic object recognition system and apply innovative technology to the computer vision recognition system to construct an automatic identification system suitable for current traffic demand and improve the stability of the traffic system.

The algorithm is improved by $\mathrm{Xu}[8]$ on the basis of convolutional neural network, and experiments are carried out on multi-source remote sensing images with different geomorphologies taken under three different weather conditions to verify the effectiveness and scalability of the improved convolutional neural network. Wang et al. [9] construct an intelligent transportation system based on neural network algorithm and combine machine vision technology to carry out intelligent monitoring and intelligent diagnosis of traffic system. Hilbert transform (HT) and relevance vector machine (RVM) are proposed by Lu et al. 
[10] to predict the sound quality of acoustical signals at power coupling mechanism of a hybrid electric vehicle (HEV). The problem of poor prediction accuracy of most SDP models is proposed by Wang et al. [11]. Wang et al. [12] conduct multi-granularity emotional block partitioning on network texts and compare the sentiment analysis under different granularities. Li and Sun [13] study the application of the radial basis function neural network model combined with the optimal segmentation algorithm in the personal loan credit rating model of banks or other financial institutions. In view of the extremely strong nonlinear processing power, self-organization, self-adaptation and learning ability of wavelet neural network (WNN), Song et al. [14] use it as the basic prediction model and use the particle swarm optimization algorithm for the slow convergence rate and local optimal problem of WNN prediction algorithm.

Acknowledgments The guest editors would like to thank Prof. John MacIntyre who is the editor in chief of Neural Computing and Applications. The guest editors would like to thank the reviewers for their high-quality reviews, which provided insightful and constructive feedback to the authors of the papers. The guest editors also would like to thank journal editor Rachel Moriarty, Deepika Sureshkumar, and Rashmi Jenna for their help on submission and publication.

\section{References}

1. Wang Y, Feng Y, Sun H (2020) Research on vehicle intelligent wireless location algorithm based on convolutional neural network. Neural Comput Appl. https://doi.org/10.1007/s00521-02004911-w

2. Zhu Z, Li D, Hu Y et al (2020) Indoor scene segmentation algorithm based on full convolutional neural network. Neural Comput Appl. https://doi.org/10.1007/s00521-020-04961-0
3. Li D, Deng L, Cai Z (2020) Research on image classification method based on convolutional neural network. Neural Comput Appl. https://doi.org/10.1007/s00521-020-04930-7

4. Chen C, Geng L, Zhou S (2020) Design and implementation of bank CRM system based on decision tree algorithm. Neural Comput Appl. https://doi.org/10.1007/s00521-020-04959-8

5. Zhang Y, Wang S, Zhao H et al (2020) CT image classification based on convolutional neural network. Neural Comput Appl. https://doi.org/10.1007/s00521-020-04933-4

6. Liu A, Zhang Y, Zhao H et al (2020) Neural network control system of cooperative robot based on genetic algorithms. Neural Comput Appl. https://doi.org/10.1007/s00521-020-04952-1

7. Li D, Deng L, Cai Z (2020) Design of traffic object recognition system based on machine learning. Neural Comput Appl. https:// doi.org/10.1007/s00521-020-04912-9

8. Xu B (2020) Improved convolutional neural network in remote sensing image classification. Neural Comput Appl. https://doi. org/10.1007/s00521-020-04931-6

9. Wang Y, Wang Q, Suo D et al (2020) Intelligent traffic monitoring and traffic diagnosis analysis based on neural network algorithm. Neural Comput Appl. https://doi.org/10.1007/s00521020-04899-3

10. Lu Y, Zuo Y, Wang $\mathrm{H}$ et al (2020) Sound quality prediction for power coupling mechanism of HEV based on CEEMD-HT and RVM. Neural Comput Appl. https://doi.org/10.1007/s00521-02004934-3

11. Wang K, Liu L, Yuan C et al (2020) Software defect prediction model based on LASSO-SVM. Neural Comput App. https://doi. org/10.1007/s00521-020-04960-1

12. Sun H, Wang G, Xia S (2020) Text tendency analysis based on multi-granularity emotional chunks and integrated learning. Neural Comput Appl. https://doi.org/10.1007/s00521-020-04901$\mathrm{y}$

13. Li X, Sun Y (2020) Application of RBF neural network optimal segmentation algorithm in credit rating. Neural Comput Appl. https://doi.org/10.1007/s00521-020-04958-9

14. Chen Q, Song Y, Zhao J (2020) Short-term traffic flow prediction based on improved wavelet neural network. Neural Comput Appl. https://doi.org/10.1007/s00521-020-04932-5

Publisher's Note Springer Nature remains neutral with regard to jurisdictional claims in published maps and institutional affiliations. 